\title{
RIESZ THEORY WITHOUT AXIOM OF CHOICE
}

\author{
ERICH MARTENSEN
}

\begin{abstract}
In this paper the Riesz theory for compact linear operators in a normed vector space is considered from the point of view of how far the axiom of choice is involved. Special attention is drawn to the theorem, by which for the operator $I-A, A$ being compact, the index vanishes and the nullspace has a closed algebraic complement. It is shown that this can be proved without making use of the axiom of choice.
\end{abstract}

Let $X$ be a normed vector space over the scalar field $\mathrm{K}$ of the real or complex numbers, and $A: X \rightarrow X$ be a compact linear operator. Then the following three theorems of the Riesz theory concerning the linear operator $S:=I-A$ are achieved by methods not using the axiom of choice (and so they were originally obtained by F. Riesz in 1918 [3]).

ThEOREM 1 (FIRST RIESZ THEOREM). The nullspace of the operator $S$ (as well as the nullspaces of all powers of $S$ ) is a finite-dimensional subspace.

THEOREM 2 (SECOND RIESZ THEOREM). The range of the operator $S$ (as well as the ranges of all powers of $S$ ) is a closed subspace.

ThEOREM 3 (THIRD RIESZ THEOREM). For the operator $S$, there exists a uniquely determined nonnegative integer $r$, called the Riesz number of $S$, satisfying the conditions ${ }^{1}$

$$
\begin{gathered}
\{0\}=N\left(S^{0}\right) \subset N\left(S^{1}\right) \subset \cdots \subset N\left(S^{r}\right)=N\left(S^{r+1}\right)=\cdots, \\
X=R\left(S^{0}\right) \supset R\left(S^{1}\right) \supset \cdots \supset R\left(S^{r}\right)=R\left(S^{r+1}\right)=\cdots, \\
X=N\left(S^{r}\right) \oplus R\left(S^{r}\right) .
\end{gathered}
$$

For the further Riesz theory, however, essential use is made of the axiom of choice: So the coincidence of the dimension of the nullspace $N(S)$ with the codimension of the range $R(S)$ is nowadays proved in a very elegant manner by the index theorem, while the existence of a closed algebraic complement to $N(S)$ is assured by means of the dual system formed by the underlying normed vector space and its (topologic) dual space [2]. In the following we will show that for these two statements the axiom of choice can be avoided. ${ }^{2}$ We start with some preparatory lemmas which will be proved here for completeness though they might be found, in one or another form, in the literature.

Received by the editors January 26, 1986. The contents of this paper have been presented to the meeting on Methods and Techniques in Mathematical Physics, held December 1-7, 1985, at the Mathematisches Forschungsinstitut Oberwolfach, Federal Republic of Germany.

1980 Mathematics Subject Classification (1985 Revision). Primary 47B05; Secondary 04A25.

${ }^{1}$ The nullspace and the range of an operator are denoted by $N(\cdot)$ and $R(\cdot)$, respectively.

${ }^{2}$ Recently Colton and Kress [1, pp. 16-22] gave an approach to the Fredholm theory for compact linear operators with respect to a dual system where the axiom of choice is not needed. This result can be reobtained in a considerably simplified manner by means of the present paper. 
LEMMA 1. For subspaces $U, V, W$ satisfying $U \subseteq W \subseteq U \oplus V$ it holds that $W=U \oplus(V \cap W)$.

Proof. By supposition, the direct sum $U \oplus V$ is defined, so $U \oplus(V \cap W)$ is defined from $U \cap(V \cap W)=(U \cap V) \cap W=\{0\} \cap W=\{0\}$. Now let $w \in W$. Then $w \in U \oplus V$, and so we have $w=u+v$ where $u \in U, v \in V$. From $U \subseteq W$ it follows that $u \in W$, further $v=w-u \in W$, and therefore $v \in V \cap W$. Hence $w=u+v \in U \oplus(V \cap W)$. On the other hand, $U \subseteq W$ and $V \cap W \subseteq W$ yield $U \oplus(V \cap W) \subseteq W$.

LEMMA 2. The projection of a normed vector space along a closed subspace into a finite-dimensional subspace forms a bounded linear operator.

ProOF. ${ }^{3}$ Let the normed vector space be represented by $X=U \oplus V$, where $U$ is a finite-dimensional subspace and $V$ is a closed subspace, respectively. Assume that the corresponding linear projection operator $P: X \rightarrow U$ is not bounded. Then there must exist a sequence $x_{n}=u_{n}+v_{n} \in X$ with $u_{n} \in U, v_{n} \in V$, which satisfies the inequality

$$
\left\|u_{n}\right\|=\left\|P x_{n}\right\|>n\left\|x_{n}\right\|=n\left\|u_{n}+v_{n}\right\| .
$$

Dividing by $\left\|u_{n}\right\|>0$ and replacing $u_{n} /\left\|u_{n}\right\|$ and $v_{n} /\left\|u_{n}\right\|$ by $u_{n} \in U$ and $v_{n} \in V$, respectively, it follows that

$$
\left\|u_{n}+v_{n}\right\|<1 / n, \quad\left\|u_{n}\right\|=1 .
$$

As $u_{n} \in U$ is now a bounded sequence in a finite-dimensional subspace, taking $u_{n}$ as a convergent sequence with a limit element $u \in U$ does not restrict our proof. From (4) we get $\|u\|=1$, hence $u \neq 0$, and $U \cap V=\{0\}$ yields $u \notin V$. On the other hand we obtain from (4)

$$
\left\|-v_{n}-u\right\|=\left\|\left(u_{n}-u\right)-\left(u_{n}+v_{n}\right)\right\|<\left\|u_{n}-u\right\|+1 / n,
$$

so $u$ turns out to be a limit element of the sequence $-v_{n} \in V$. Finally using that $V$ is closed, we are led to the contradiction that $u \in V$.

LEMMA 3. In a normed vector space, the direct sum of a finite-dimensional subspace and a closed subspace forms a closed subspace.

PROOF. Let $X$ be a normed vector space containing a finite-dimensional subspace $U$ and a closed subspace $V$ for which the direct sum $U \oplus V$ is defined. As $U \oplus V \subseteq X$ itself forms a normed vector space to which Lemma 2 may be applied, we have a bounded linear projection $P: U \oplus V \rightarrow U$, carried out along $V$. Now let $x_{n} \in U \oplus V$ be an arbitrarily chosen convergent sequence with limit element $x \in X$. Then together with $x_{n}=u_{n}+v_{n}$, where $u_{n} \in U, v_{n} \in V$, it follows that

$$
\left\|u_{m}-u_{n}\right\|=\left\|P x_{m}-P x_{n}\right\|=\left\|P\left(x_{m}-x_{n}\right)\right\| \leq\|P\|\left\|x_{m}-x_{n}\right\| .
$$

Thus $u_{n}$ forms a Cauchy sequence within the finite-dimensional subspace $U$ and therefore converges to a limit element $u \in U$. Furthermore, the sequence $v_{n}=$ $x_{n}-u_{n} \in V$ is also convergent, and taking into account that $V$ is closed, it has a limit element $v \in V$. If we now let $n \rightarrow \infty$ in $x_{n}=u_{n}+v_{n}$, the assertion is obtained from $x=u+v \in U \oplus V$.

\footnotetext{
${ }^{3}$ Simplified version of the proofs contained in $[1$, pp. 15-16] and $[2$, pp. $45,114,124-125]$.
} 
THEOREM 4 (FOURTH RIESZ THEOREM) ${ }^{4}$ For the range $R(S)$, there exists a finite-dimensional algebraic complement with the same dimension as the nullspace $N(S)$; furthermore for the nullspace $N(S)$, there exists a closed algebraic complement.

Proof. Only needed for $r>0$. As the (finite-dimensional) nullspace $N(S)$ is a subspace of the (finite-dimensional) subspace $N\left(S^{r}\right)$, a finite-dimensional subspace $U_{0} \subseteq N\left(S^{r}\right)$ satisfying

$$
N\left(S^{r}\right)=N(S) \oplus U_{0}
$$

can be obtained. Now with regard to Theorem 2 and (3), if the closed subspace $R\left(S^{r}\right)$ is directly added to both sides of (5) and Lemma 3 is observed, then the direct sum $U_{0} \oplus R\left(S^{r}\right)$ forms a closed algebraic complement to $N(S)$. Furthermore, Lemma 2 gives us a bounded linear projection $P: X \rightarrow N(S)$.

From $r>0$ and (1) it follows that the nullspace $N(S)$ must have a positive dimension $m$. Let $a_{1}, \ldots, a_{m}$ be a basis for $N(S)$. Further, let $\varphi_{0}^{i}: N(S) \rightarrow \mathrm{K}$ be the bounded linear functional which to every element of $N(S)$ adjoins its scalar component with respect to the basis element $a_{i} \in N(S), i=1, \ldots, m$. Consequently, all the $m$ products (compositions)

$$
\varphi^{i}:=\varphi_{0}^{i} P: X \rightarrow \mathrm{K}, \quad i=1, \ldots, m,
$$

are also bounded linear functionals. It obviously holds that

$$
\begin{aligned}
P x & =\sum_{i=1}^{m}\left(\varphi_{0}^{i}(P x)\right) a_{i}=\sum_{i=1}^{m}\left(\varphi^{i} x\right) a_{i}, \quad x \in X, \\
\varphi^{i} a_{k} & =\varphi_{0}^{i}\left(P a_{k}\right)=\varphi_{0}^{i} a_{k}=\delta_{k}^{i}, \quad i, k=1, \ldots, m .
\end{aligned}
$$

From $r>0$, (2), (3), and Lemma 1 we get

$$
R(S)=R\left(S^{r}\right) \oplus\left(N\left(S^{r}\right) \cap R(S)\right) .
$$

As $N\left(S^{r}\right) \cap R(S)$ is a subspace of the (finite-dimensional) subspace $N\left(S^{r}\right)$, there is a finite-dimensional subspace $U \subseteq N\left(S^{r}\right)$ such that

$$
N\left(S^{r}\right)=\left(N\left(S^{r}\right) \cap R(S)\right) \oplus U .
$$

If, with regard to (3) and (8), the subspace $R\left(S^{r}\right)$ is added directly to both sides of (9), then it follows that

$$
X=R(S) \oplus U .
$$

Thus $U$ turns out to be an algebraic complement to $R(S)$ which because of $r>0$ and (2) must have a positive dimension $n$. Let $u_{1}, \ldots, u_{n}$ be a basis for $U$.

Next we introduce a linear operator $\tilde{S}: X \rightarrow X$ by its values

$$
\tilde{S} x:=S x+\sum_{i=1}^{\min \{m, n\}}\left(\varphi^{i} x\right) u_{i}, \quad x \in X .
$$

\footnotetext{
${ }^{4}$ This notation may be considered as historically not quite justified.
} 
The operator $\tilde{S}$ differs from $S$ by an additive finite-dimensional linear operator which because of

$\left\|\sum_{i=1}^{\min \{m, n\}}\left(\varphi^{i} x\right) u_{i}\right\| \leq \sum_{i=1}^{\min \{m, n\}}\left|\varphi^{i} x\right|\left\|u_{i}\right\| \leq\left\{\sum_{i=1}^{\min \{m, n\}}\left\|\varphi^{i}\right\|\left\|u_{i}\right\|\right\}\|x\|, \quad x \in X$,

is bounded and therefore compact. Together with $S=I-A$ we get $\tilde{S}=I-\tilde{A}$ with a compact linear operator $\tilde{A}: X \rightarrow X$; so the theory as far as it has been developed above may be applied to $\tilde{S}$. In order to finish the proof by showing that $m=n$ we shall, however, only make use of the fact that by Theorem 3 , injectivity and surjectivity of the operator $\tilde{S}$ correspond to each other.

Assume that $m<n$. Let $x \in N(\tilde{S})$, so (11) yields

$$
\tilde{S} x=S x+\sum_{i=1}^{m}\left(\varphi^{i} x\right) u_{i}=0 .
$$

As the nullelement $0 \in X$ is decomposed here by means of the direct sum (10) and the basis elements $u_{1}, \ldots, u_{m} \in U$ are linearly independent, it follows that

$$
x \in N(S), \quad \varphi^{i} x=0, \quad i=1, \ldots, m,
$$

and further together with (6) that $x=P x=0$. So $\tilde{S}$ turns out to be injective and from this to be also surjective. Thus with regard to (11), there must exist an element $x_{0} \in X$ satisfying

$$
\tilde{S} x_{0}-u_{m+1}=S x_{0}+\sum_{i=1}^{m}\left(\varphi^{i} x_{0}\right) u_{i}-u_{m+1}=0 .
$$

Again we have a decomposition of the nullelement $0 \in X$ by means of (10), and so we get the contradiction that the basis elements $u_{1}, \ldots, u_{m}, u_{m+1} \in U$ are linearly dependent.

Assume that $m>n$. From (7) and (11) we conclude

$$
\tilde{S} a_{k}=S a_{k}+\sum_{i=1}^{n}\left(\varphi^{i} a_{k}\right) u_{i}=\sum_{i=1}^{n} \delta_{k}^{i} u_{i}= \begin{cases}u_{k}, & k=1, \ldots, n \\ 0, & k=n+1\end{cases}
$$

Now let an element $x \in X$ be arbitrarily chosen. Using its decomposition with regard to (10) and referring to (11) and (12), then for some element $x_{0} \in X$ and some scalars $\lambda^{1}, \ldots, \lambda^{n} \in \mathbf{K}$ we are led to the representation

$$
x=S x_{0}+\sum_{i=1}^{n} \lambda^{i} u_{i}=\tilde{S} x_{0}+\sum_{i=1}^{n}\left(\lambda^{i}-\varphi^{i} x_{0}\right) u_{i}=\tilde{S}\left\{x_{0}+\sum_{i=1}^{n}\left(\lambda^{i}-\varphi^{i} x_{0}\right) a_{i}\right\} .
$$

Hence $\tilde{S}$ is surjective and therefore also injective. Consequently, since the basis element $a_{n+1} \in N(S)$ differs from the nullelement, it must be $\tilde{S} a_{n+1} \neq 0$. But this contradicts (12).

REMARK. As can be seen for $r>0$ from the foregoing proof and as it trivially holds for $r=0$, the finite-dimensional algebraic complement to $R(S)$ can be obtained as a subspace of $N\left(S^{r}\right)$, while the closed algebraic complement to $N(S)$ can be found as the direct sum of a subspace of $N\left(S^{r}\right)$ with $R\left(S^{r}\right)$. 


\section{REFERENCES}

1. D. Colton and R. Kress, Integral equation methods in scattering theory, Wiley, New York, 1983.

2. H. Heuser, Funktionalanalysis, Teubner, Stuttgart, 1975.

3. F. Riesz, Über lineare Funktionalgleichungen, Acta Math. 41 (1918), 71-98.

Mathematisches INSTItUT II, UNIVERSität KaRlsRuhe, 7500 KaRlsRUhe 1 , FEDERAL REPUBLIC OF GERMANY 